\title{
Candidates for non-baryonic dark matter
}

\author{
Nicolao Fornengo ${ }^{\mathrm{a}}$ \\ ${ }^{\text {a}}$ Dipartimento di Fisica Teorica, Università di Torino and INFN, Sezione di Torino \\ via P. Giuria 1, I-10125 Torino, Italy
}

This report is a brief review of the efforts to explain the nature of non-baryonic dark matter and of the studies devoted to the search for relic particles. Among the different dark matter candidates, special attention is devoted to relic neutralinos, by giving an overview of the recent calculations of its relic abundance and detection rates in a wide variety of supersymmetric schemes.

\section{Evidence of darkness}

The presence of large amounts of non-luminous components in the Universe has been identified along the years by different means and on different scales: on the galactic scale, the flatness of the rotational curves of many galaxies indicates a dark component which is presumably distributed as a halo around the galaxies [1]; clusters points toward a sizeable contribution of unseen matter distributed around and between galaxies [2]; more recently, on cosmological scales, the combination of the results on high-redshift supernovae 3 and on the anisotropies of the cosmic microwave background radiation 4 is pointing toward a flat Universe whose energy density is dominated by a dark vacuum component (cosmological constant, quintessence) together with a sizeable dark component of matter. In terms of the density parameter $\Omega$, the current view can be summarized as follows [5]: the total amount of matter/energy of the Universe is $\Omega_{\text {tot }}=1.02 \pm_{0.05}^{0.06}$, and this is composed of a matter component $\Omega_{\mathrm{M}}=0.31 \pm_{0.12}^{0.13}$ and a vacuum-energy component $\Omega_{\Lambda}=0.71 \pm 0.11$. Even though the actual numbers vary a little depending on the priors of the statistical analyses, the clear indication of the latest data is that the Universe is strongly dominated by dark (and unknown) components. In fact the numbers above cannot be reconciled with a Universe made only of standard components: from primordial nucleosynthesis studies, baryons can contribute only at the level of $\Omega_{\mathrm{b}}=0.037 \pm 0.011$ [6], while luminous matter is known to provide only a contribution of order $\Omega_{\text {lum }} \sim 0.003[7]$. We are therefore facing the presence of at least three dark components in the Universe: dark baryons, dark non-baryonic matter and dark energy. The existence of both dark (relativistic or non-relativistic) exotic matter and dark energy asks for extension of the standard model of fundamental interactions, since no known particle or field can explain either of these components.

In this review, we will discuss the current status of the non-baryonic dark matter problem. For reviews on dark baryons and dark energy, see Refs. 88,97. We will discuss the efforts to explain the amount of dark matter in the Universe, which we summarize as:

$0.05 \lesssim \Omega_{\mathrm{M}} h^{2} \lesssim 0.3$

and the studies related to the searches for dark matter particles.

\section{Particle candidates to dark matter}

Dark matter candidates must be looked for in schemes beyond the standard model. The interesting feature of many of these models is that they are extensions whose primary motivation is not related to dark matter: in spite of this, they contain particles which have the right properties to act as dark matter. This is especially true for supersymmetric theories, which are the ones with richer phenomenology for dark matter, but interesting candidates may also be found in theories which do not involve supersymmetry. 
Among the non-supersymmetric candidates, the massive neutrino and the axion are the most direct possibilities.

A light massive neutrino represents the simplest extension of the standard model. The recent results on the atmospheric [10,11] and solar [11.12] anomalies strongly indicate that neutrinos do possess a mass: a consequence of this is that neutrinos could also compose at least a fraction of dark matter. From the atmospheric neutrino analyses [10], we deduce that neutrinos can provide $\Omega_{\nu} h^{2} \gtrsim 6 \cdot 10^{-4}$, but very likely they cannot contribute more than $\Omega_{\nu} h^{2} \sim 0.05$ in order not to spoil the process of structure formation. Therefore, a massive neutrino can only be (and very likely is) present as a sub-dominant dark matter component. Although less economic from the point of view of particle physics, it is also possible to have very massive neutrinos $\left(m_{\nu} \gtrsim 100 \mathrm{GeV}\right)$ which again would contribute only partially, at the level of $\Omega_{\nu} h^{2} \sim 10^{-3}-10^{-2}$ [13]. Sterile neutrinos with masses in the $\mathrm{keV}$ range and mixing angles of the order of $10^{-10}$ rad may play a role, since they may provide $\Omega_{\nu} h^{2} \sim 0.1-0.2$ [14, and therefore explain the dark matter amount of Eq.(11).

Axions arise in models where the strong CP problem is solved by the Peccei-Quinn mechanism. They may be produced in the early Universe by means of different mechanisms, either thermally or non-thermally (misalignment of the axionic field, decay of axionic strings) [15]. The current limits [16] from axion searches and cosmology constrain this particle to have either a mass around $10 \mathrm{eV}$ or in the mass range $10^{-6}$ $10^{-2} \mathrm{eV}$. In both cases, axions can solve the dark matter problem stated in Eq.(1), but they can also be a sub-dominant component.

Supersymmetry with conserved R-parity offers a wide variety of dark matter candidates. The nature of the relic particle depends somehow on the way supersymmetry is broken. Gravity-mediated models predict as dark matter particle either the neutralino or the sneutrino [17]. The neutralino may be the dark matter candidate also in anomaly-mediated models. In gauge mediatedmodels the role of dark matter may be played by the gravitino [18] or by messenger fields [19]. In supersymmetric models where the strong CP problem is solved by the axion, its supersymmetric partner, the axino [20] is also present and can act as dark matter. Other possibilities that have been proposed are stable non-topological solitons, like the $Q$-balls 21], or heavy non-thermal relics 22. The most interesting candidate, and the one which has been more deeply investigated, is the neutralino, and in the following Sections we will concentrate our discussion on this particle. For the other supersymmetric candidates, we refer to the quoted references and the references quoted therein.

Finally, among other candidates which have been proposed, not necessarily related to supersymmetry, we finally recall mirror particles [23] or some type of scalar fields [24].

From the above discussion on particle dark matter candidates, a fact clearly arises: nonbaryonic dark matter may naturally be multicomponent. An example of this occurs, for instance, in models where the CP problem is solved by the axion and the hierarchy problem is explained by low-energy supersymmetry. In this case, the axion and the axino or the neutralino are all dark matter components, plus the massive neutrino which nowadays appears to be an unavoidable dark matter component. Which one would turn out to be the dominant candidate would depend on the characteristics of the specific model which one can build. But the possibility of multi-component dark matter is interesting, because the detectability of a specific candidate is not in general related to the fact that this particle is the dominant component of dark matter. Usually, at least for candidates like the neutralino in standard cosmology, it turns out that it is easier to detect a relic particle which is sub-dominant [25]. This is related to the fact that detection rates rely on the cross sections of the processes of scattering or annihilation, while on the contrary the relic abundance is inversely proportional to the annihilation cross section. This roughly induces an anti-correlation between detection rates and cosmological abundance: therefore large detection rates are often related to low relic abundances. It is not trivial to have a relic particle 
which is at the same time a dominant dark matter component and potentially detectable. Clearly this last situation is the most appealing one.

As for the most studied candidate which is the neutralino, in the following it will be shown that this situation can occur in suitable classes of supersymmetric models. This is one of the properties that makes the neutralino a very appealing candidate.

\section{Supersymmetry and dark matter: the neutralino}

The existence of a relic particle in supersymmetric theories arises from the conservation of a symmetry, $R$-parity, which prevents the lightest of all the superpartners from decaying. The nature and the properties of this particle depend on the way supersymmetry is broken, and we have already seen that the neutralino can be the dark matter candidate in models where supersymmetry is broken through gravity- (or anomaly-) mediated mechanisms. The actual implementation of a specific supersymmetric scheme depends on a number of assumptions on the structure of the model and on the relations among its parameters. This induces a large variability of the phenomenology of neutralino dark matter. In this Section we briefly recall the supersymmetric models which have been mostly studied for neutralino dark matter, while in the next Sections we will give a few examples of calculations of relic abundance and detection rates in some of these schemes. For details concerning the models and their implementations for neutralino dark matter calculations, we refer to the list of references provided below and references quoted therein.

The simplest and most direct implementation of supersymmetry is represented by the minimal supergravity (mSUGRA) scheme 26 42, where, in addition to requiring gauge coupling constant unification at the GUT scale, also all the mass parameters in the supersymmetric breaking sector are universal at the same GUT scale. The lowenergy sector of the model is obtained by evolving all the parameters through renormalization group equations (RGE): this process also induces the breaking of the electroweak (EW) symmetry in a radiative way (rEWSB). This model is very predictive, since it relies only on four free parameters, but at the same time it induces a very constrained phenomenology at low-energy. It also appears to be quite sensitive to some standard model parameters, like the mass of the top and bottom quarks $\left(m_{t}\right.$ and $\left.m_{b}\right)$ and the strong coupling constant $\alpha_{s}$.

A more relaxed implementation of this supersymmetric scheme is offered by non-universal supergravity (nuSUGRA) [26 30,35, 41, 43, 45, where some of the unification conditions at the GUT scale are relaxed: non-universality has been studied in the Higgs, in the sfermion and in the gaugino sectors.

Specific patterns of non-universality may be originated through mechanisms which involve effects of extra-dimensions, like in $D$-brane 46,48 , 49] and string models [49]. These models also may be very predictive, with very few free parameters, but the relations among them is different from what is postulated in mSUGRA models.

It has also been realized that unification conditions, both for gauge couplings and/or mass parameters, may occur at scales which are different from the standard GUT scale at about $10^{16} \mathrm{GeV}$. This unification scale may be lower than the usual GUT scale (intermediate unification scale models) [50.51], and this induces a modification of neutralino phenomenology at low energy.

A different approach is offered by the lowenergy supersymmetric model (effMSSM) 22,,29, 30, 39,52 70, defined directly at the EW scale, which is where the phenomenology of neutralino dark matter is actually studied. Also in this case we have to make assumptions in order to reduce the number of free parameters to a manageable number. These assumptions must be mild enough not to represent an arbitrary over-constraint on the model, and all the relevant parameters at the EW scale must be represented. It is possible in this case to work with six or seven free parameters.

Other models which have been discussed in the literature in connection with neutralino dark matter are dilaton domination models |71], models with $C P$-violation $[72$ and anomaly mediated models 773 . 
The parameter space of all the models is constrained by a number of relevant experimental bounds which mostly come from accelerator physics. Negative searches of supersymmetry at LEP [74] and Tevatron [75] are currently constraining charginos to be heavier than about 105 $\mathrm{GeV}$ and sleptons and squarks to be heavier than 90-100 GeV. The bounds from LEP on the lightest supersymmetric Higgs boson $h$ indicate that its mass is larger than $90 \mathrm{GeV}$ for small coupling of $h$ with $b$-quarks, while for intermediate and large values of this coupling the bound on the mass is about $114 \mathrm{GeV}$ 74]. Tevatron further constrains the mass of $h$ in some sectors of the supersymmetric parameter space (namely, large values of $\tan \beta$, the ratio of the vev's of the two neutral higgses) 75. Finally, an important constraint for all supersymmetric models comes from the measured values of the branching ratio of the radiative decay $b \rightarrow s+\gamma$, which, in addition to the standard model terms, can get sizeable contributions from supersymmetric loops $[76$. Notice that the requirement that the neutralino is a dark matter particle, implies that also cosmology imposes a limit on supersymmetric models: in fact, the relic abundance of neutralinos, which can be calculated in a given supersymmetric scheme, cannot exceed the upper limit of Eq.(11).

At present, there is no direct indication that supersymmetry has been found in Nature. However, we just want to mention a few recent results which have been considered as possible hints of supersymmetry. The first is the excess of signallike events in the Higgs searches at LEP: an effect of about $2-\sigma$ for a Higgs mass around 115 $\mathrm{GeV}$ has been reported [77]. This light higgs may be interpreted as indicative of a supersymmetric higgs. Another indication for physics beyond the standard model comes from a global fit to the precision electroweak data: the analysis shows that the standard model is acceptable with a low (around 4\%) confidence level [78]. The agreement with the data improves considerably if low-energy supersymmetry is included in the analysis 78 ].

In the last year another anomaly raised a lot of attention: a possible deviation between the calculated and the measured value of the anomalous magnetic moment of the muon. However, recent

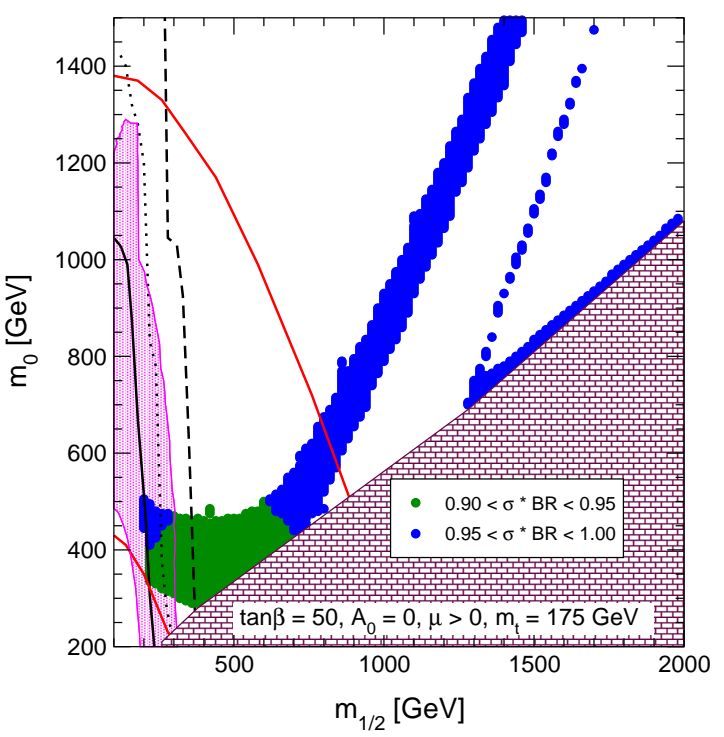

Figure 1. Neutralino relic abundance in a mSUGRA scheme, for $\tan \beta=50$ and $m_{t}=175$ $\mathrm{GeV}$ [32]. Inside the dark (green and blue) bands: $0.1 \leq \Omega_{\chi} h^{2} \leq 0.3$.

theoretical re-evaluations have shown that the evidence of deviation is very weak 79, and therefore instead of indicating a possible presence of supersymmetry, the results on the muon anomalous magnetic moment should be regarded as imposing bounds on the supersymmetric parameter space, as is the case for $b \rightarrow s+\gamma$.

\section{Neutralino relic abundance}

Neutralino relic abundance, like also neutralino detection rates, is sensitive to the specific supersymmetric model which is employed. In fig. 1 we show an example, taken from Ref. 32|, which refers to a mSUGRA scheme 226, 28, 30 32, 36, 47 in the large $\tan \beta$ regime. The regions of the parameter space shown in the figure as dark (blue and green) bands entail a relic neutralino with relic abundance in the range $0.1 \leq \Omega_{\chi} h^{2} \leq 0.3$. Therefore, in that sector of the parameter space the neutralino represents the dominant dark matter component since it is able to fulfill the cosmological requirement of Eq.(11). Instead, in the region which is between the thick and the 
thin (blue) bands, neutralino relic abundance is smaller than 0.1 , and in a part of this region it falls below the lower limit of Eq.(11): in this last case, neutralino cannot account for all the dark matter in the Universe. On the contrary, in the other two large white regions of fig. 1, $\Omega_{\chi} h^{2}>0.3$ : these regions of the mSUGRA parameter space have to be considered as excluded by the requirement that neutralino relic abundance does not get in conflict with the experimental determination of the amount of dark matter in the Universe. In the same parameter space of fig. 1, another region has been identified where $\Omega_{\chi} h^{2}$ can fall in the range of Eq.(11): this can occur at large $m_{0} / m_{1 / 2}$ 26, 34, a region which has been also called as "focus point model" ( $m_{0}$ is the common soft scalar mass parameter at the GUT scale and $m_{1 / 2}$ is the common gaugino mass at the GUT scale).

The situation shown if Fig.1 can change substantially if one explores other sectors of the mSUGRA parameter space, like for instance small values of $\tan \beta$, where the region in which neutralino is the dominant component is quite constrained to values of $m_{0}$ and $m_{1 / 2}$ which do not exceed a few hundreds of $\mathrm{GeV}$, except in very narrow corridors where coannihilation between neutralinos and staus occur [32].

It has to be noticed that mSUGRA models turn out to be somewhat sensitive to the standard model parameters $m_{t}, m_{b}$ and $\alpha_{s}$ and to the details of radiative effects (radiative corrections, radiative electroweak symmetry breaking). It is therefore difficult to derive definite conclusions on which are the intervals of the mSUGRA parameters where neutralino is a dominant dark matter component (except for small values of $\tan \beta$ ).

The effects of non-universality [26, 28, 30, 43, 45, 46] affect the low-energy sector of the theory through changes in the RGE and in the occurrence of rEWSB. This has the effect of modifying neutralino couplings and the mass spectrum of supersymmetric particles, with the consequence of modifying neutralino relic abundance and the cosmologically relevant regions in the nuSUGRA parameter space. Quite relaxed relic abundance regions are also obtained in the low-energy realization of supersymmetry, the effMSSM [25,30.
$39,57,59,62$.

\section{Relic neutralino searches}

Neutralino relic particles can be searched for by means of different techniques. Basically, the detection methods are of two types: direct detection, which relies on the possibility to directly measure the interaction of neutralinos with a detector in the laboratory, or indirect detection, which tries to identify products of neutralino annihilation.

Dark matter particles are supposed to be bounded to the Galaxy, since they are part of the dark halo which is responsible for the observed flatness of the rotational curves. Therefore the best place to look for relic neutralinos is the halo of our Galaxy. The dynamics and distribution of these particles are described by a matter- and a velocity- distribution function, about which there are still large uncertainties.

The distribution in space of these bounded dark particles can be smooth and non-singular at the galactic center, but numerical simulations tend to indicate a cuspy behaviour toward the center of the Galaxy as well as a clumpy distribution of dark matter in the halo. Detection rates which depend on annihilation processes which occur in the galactic halo are therefore strongly affected by this uncertainty in the matter distribution. On the contrary, detection rates which rely on local properties, like direct detection, are not very much sensitive on how dark matter is distributed in the halo. In this case, the relevant parameter is the total local dark matter density $\rho_{l}=(0.2 \div 0.7)$ $\mathrm{GeV} \mathrm{cm} \mathrm{cm}^{-3} 29$. Neutralinos can account for all or part of this amount, depending whether they are or not the dominant dark matter component. Therefore, the local neutralino matter density is: $\rho_{\chi}=\xi \rho_{l}$, where $\xi \leq 1$ [25].

The velocity distribution of dark matter particles in the halo is even more uncertain than the matter distribution, since it is difficult to constrain the way these dark particles move in the halo. The usual assumption is to consider an isotropic Maxwell-Boltzmann (MB) distribution, as seen in the galactic rest frame. The relevant parameters in this case are only two: the local rotational velocity $v_{0}=(220 \pm 50) \mathrm{km} \mathrm{s}^{-1}$ and the 
escape velocity $v_{\text {esc }}=(450 \div 650) \mathrm{km} \mathrm{s}^{-1} 54$. However, the velocity distribution can be quite different from the simple MB form: it can still be isotropic but non-maxwellian, as well as nonisotropic. Moreover, the halo can be affected by some amount of rotation. The way dark matter particles behave in velocity space affects mostly direct detection, which relies on the energy transfered from the dark matter particles to a detector.

Proposals of looking for extra-galactic neutralino dark matter have also been put forward. One possibility is to look for energetic gamma rays from neutralino annihilation in dense extragalactic systems, like M87 and local dwarfs spheroidal galaxies [69]: in order to have potentially detectable signals, clumpiness is needed. Another possibility is the direct detection of dark matter particles coming from outside our Galaxy [70]: these particles have a much lower flux than galactic neutralinos, but they posses some typical features, like an essentially unique velocity and very few flight directions, which can help in discriminating the extra-galactic particles from the ones bounded to the Galaxy.

\subsection{Direct detection}

Direct detection relies on the scattering of dark matter particles off the nuclei of a lowbackground detector. This method is sensitive to the local properties of the neutralinos in the halo, i.e. its local abundance $\rho_{\chi}$ and its local velocity distribution, and depends on the neutralino-nucleus scattering cross section, which is usually dominated by the coherent interaction. The detection rate is proportional to the product $\left[\rho_{\chi} \times \sigma_{\text {scalar }}^{\text {(nucleon) }}\right]$ for any given velocity distribution [29]. The experimental results on direct detection are reviewed in Ref. [80]. We only recall that the current sensitivity of direct detection experiments can be quoted in the following ranges for neutralino-nucleon cross section and mass: $4 \cdot 10^{-9}$ nbarn $\lesssim \xi \sigma_{\text {scalar }}^{\text {(nucleon) }} \lesssim$ few $\cdot 10^{-8}$ nbarn and $30 \mathrm{GeV} \lesssim m_{\chi} \lesssim 200 \mathrm{GeV}$ [29]. In the above, we have considered the uncertainties in the local values of the dark matter density and some uncertainties in the halo models, discussed in the previous Section. We recall that the quantity $\xi$ measures the fraction of local dark matter to be

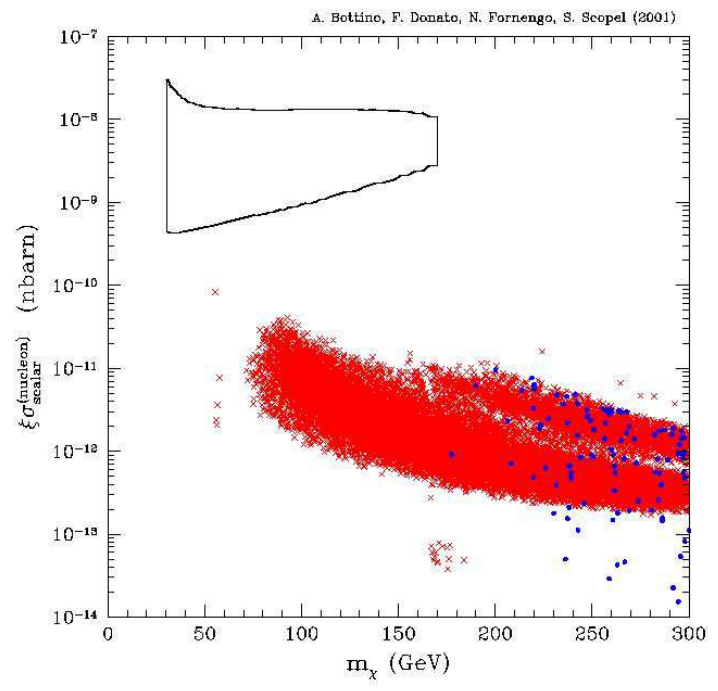

Figure 2. Relevant direct detection cross section times the fractional amount of neutralino dark matter in a mSUGRA scheme, with $m_{t}, m_{b}$ and $\alpha_{s}$ fixed at their central values. The (red) crosses refer to dominant neutralino dark matter, while the (blue) dots refer to sub-dominant neutralinos $\left(\Omega_{\chi} h^{2}<0.05\right)$.

ascribed to the neutralino $(\xi \leq 1)$ [25]. This experimental sensitivity of direct detection is shown in figs. 2 - 2 as a closed region.

The contour which is reported in these figures is actually the DAMA/NaI region which is obtained when the annual modulation effect observed by the DAMA Collaboration is interpreted as due to a dark matter particle 81. The presence of an annual modulation in the low-energy recoil spectrum is in fact a distinctive signature of direct detection searches 82.

Figs. 2 and 3 show the comparison between the experimental results and the theoretical calculations in the mSUGRA scheme. The quantity $\xi$, which determines whether the neutralino is a dominant or sub-dominant component, is calculated according to its relic abundance as: $\xi=$ $\min \left(1, \Omega_{\chi} h^{2} / 0.05\right)$ 25]. The comparison of the two figures shows that in a constrained mSUGRA [26, 28 30,33, 35, 37, 40,48] scheme the calculation are very sensitive also to standard model param- 


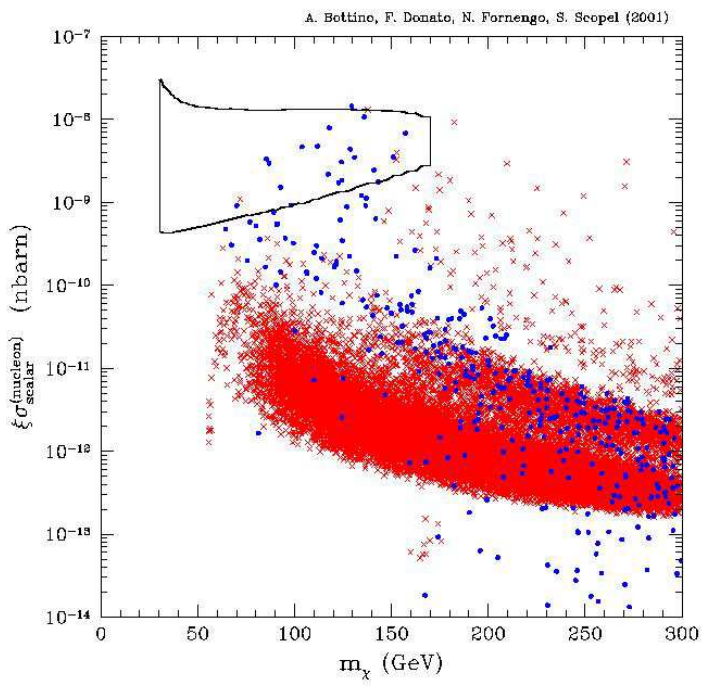

Figure 3. The same as in fig.2, except that $m_{t}$, $m_{b}$ and $\alpha_{s}$ are varied inside their $2-\sigma$ allowed intervals.

eters [30]: in fig.2 $m_{t}, m_{b}$ and $\alpha_{s}$ are fixed at their own experimental central values, while in fig. 3 these quantities are allowed to vary inside their $2-\sigma$ intervals. We also comment that, especially in strict mSUGRA models, radiative effects are quite critical, especially in the higgs sector where light higgses $\left(m_{h} \sim 90-100 \mathrm{GeV}\right)$, not in conflict with the experimental bounds from LEP, can be obtained for large values of $\tan \beta$ 30,83 and this can induce relatively large values for $\xi \sigma_{\text {scalar }}^{\text {(nucleon) }}$ [29, 30]. Also the signal-like LEP higgs events may be compatible with interesting neutralino dark matter phenomenology for direct detection [30].

Non-universal models [26,28, 30, 13, 48] are naturally more relaxed and values of $\sigma_{\text {scalar }}^{\text {(nucleon) at }}$ the level of current sensitivies are more easily obtained, both for a dominant and a subdominant relic neutralino: an example is shown in fig.4. Also in D-brane models 48,49,51, and even more easily in the effMSSM $25.29,30,39,52$, 53.60], cross sections large enough to be probed by direct detection are obtained. Ax example for a D-brane model with intermediate unification scale is shown in fig. .

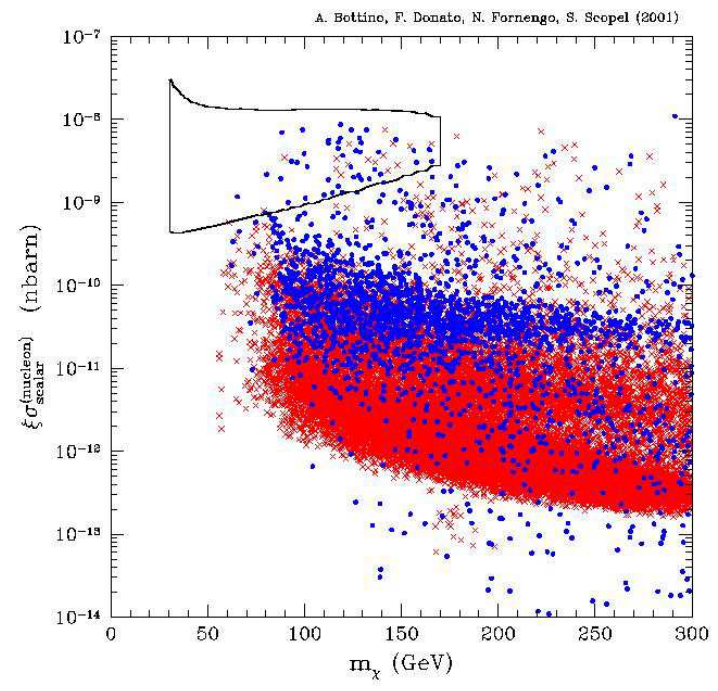

Figure 4. The same as in fig. 3 , for a nuSUGRA scheme.

The question whether current direct detection sensitivities are probing dominant or subdominant relic neutralinos may be answered in terms of the plot shown if fig. 6 , which translates directly in terms of astrophysical and cosmological quantities the direct detection results [25, 29, 53, 54, 84]. By considering the current interval of sensitivities on the quantity $\left[\rho_{\chi} \times \sigma_{\text {scalar }}^{(\text {nuclen })}\right]$, the calculation of $\sigma_{\text {scalar }}^{\text {(nucleon) }}$ allows us to determine the values of $\rho_{\chi}$ which are required for each supersymmetric configuration in order to provide a detectable signal (for details see for instance Ref. [29] ). Fig.6 shows the calculated values of $\rho_{\chi}$ vs. the neutralino relic abundance, for the effMSSM. We see that a fraction of supersymmetric models overlap with the region of main cosmological and astrophysical interest: $0.05 \lesssim \Omega_{\chi} h^{2} \lesssim 0.3$ and $0.2 \leq \rho_{\chi} /\left(\mathrm{GeVcm}^{-3}\right) \leq 0.7$. For points in this region, the neutralino is the dominant component of dark matter both in the Universe and at the galactic level. For points which fall inside the band delimited by the slant dot-dashed lines, the neutralino would provide only a fraction of the cold dark matter both at the level of local density and at the level of the average $\Omega$, a situation which would be possible if the neutralino is not 


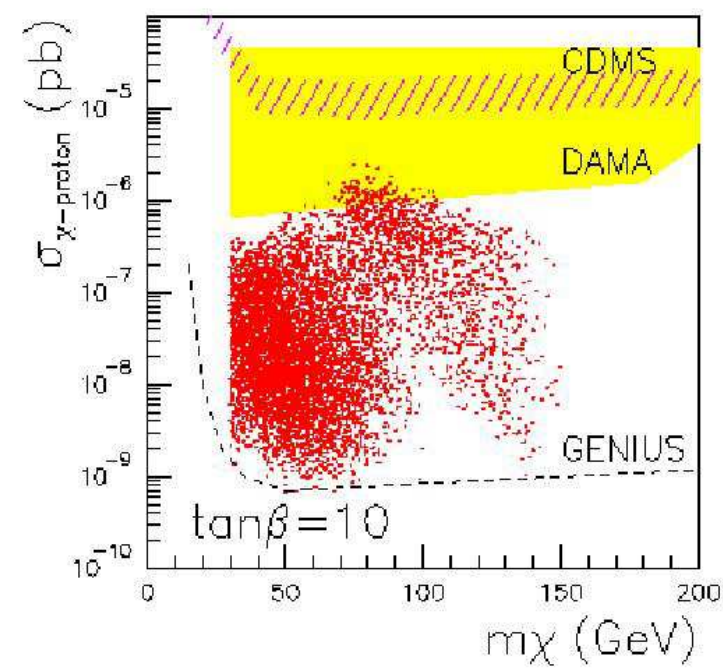

Figure 5. Relevant direct detection cross section in a D-brane model with an intermediate unification scale at $10^{12} \mathrm{GeV}$ 51].

the unique cold dark matter particle component. On the other hand, configurations above the upper dot-dashed line and below the upper horizontal solid line would imply a stronger clustering of neutralinos in our halo as compared to their average distribution in the Universe. This situation may be considered unlikely, since in this case neutralinos could fulfill the experimental range for $\rho_{\chi}$, but they would contribute only a small fraction to the cosmological cold dark matter content. Finally, configurations above the upper horizontal line are incompatible with the upper limit on the local density of dark matter in our Galaxy.

\subsection{Indirect detection}

Indirect detection relies on the possibility to identify signals which are originated by neutralino self-annihilations. There are two basic situations: annihilation inside the Earth or the Sun, or annihilation in the galactic halo.

The first type of signal, due to neutralino annihilation taking place in celestial bodies (Earth or Sun) where the neutralinos have been gravitationally captured and accumulated, is a neutrino flux, which can be detected in a neutrino telescope as a flux of up-going muons [27, 41, 52, 55, 58,64. 64 .

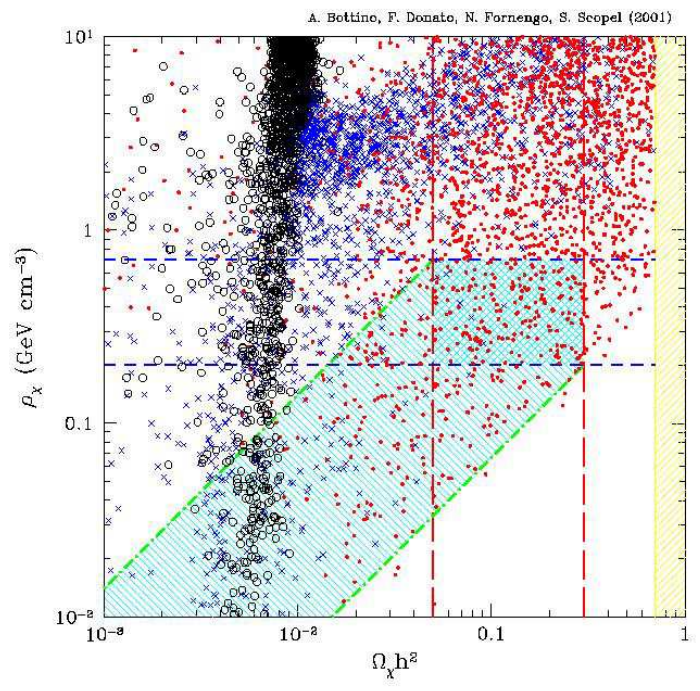

Figure 6. Neutralino local density $\rho_{\chi}$ derived by requiring that $\left[\rho_{\chi} \sigma_{\text {scalar }}^{\text {(nucleon) }}\right]$ is at the level of the current experimental sensitivity in direct detection, plotted vs. the neutralino relic abundance $\Omega_{\chi} h^{2}$ in the effMSSM.

Since the process relevant for accumulation of neutralino is capture, which relies on neutralino scattering off the nuclei of the Earth and the Sun, this detection technique is sensitive only to local properties of the Galaxy, like direct detection. In figs. 7 and 8 we show the flux of upgoing muons calculated in the effMSSM for the signal coming from the Earth and the Sun, respectively 52]. The supersymmetric configurations which are shown are only the ones which are currently at the level of being explored by direct detection and the upper limit on the upgoing muon flux from MACRO is shown as a solid line. We can see that indirect detection at neutrino telescopes is partially competitive with direct detection, although a large fraction of configurations which are explored by direct detection require more sensitive neutrino telescopes in order to be probed.

Neutralino annihilations can take place also directly in the galactic halo. In this case the signals can consist in: a diffuse neutrino flux 65; a diffuse gamma-ray flux or a gamma-ray line 62, 63, 65]; exotic components in cosmic rays: 


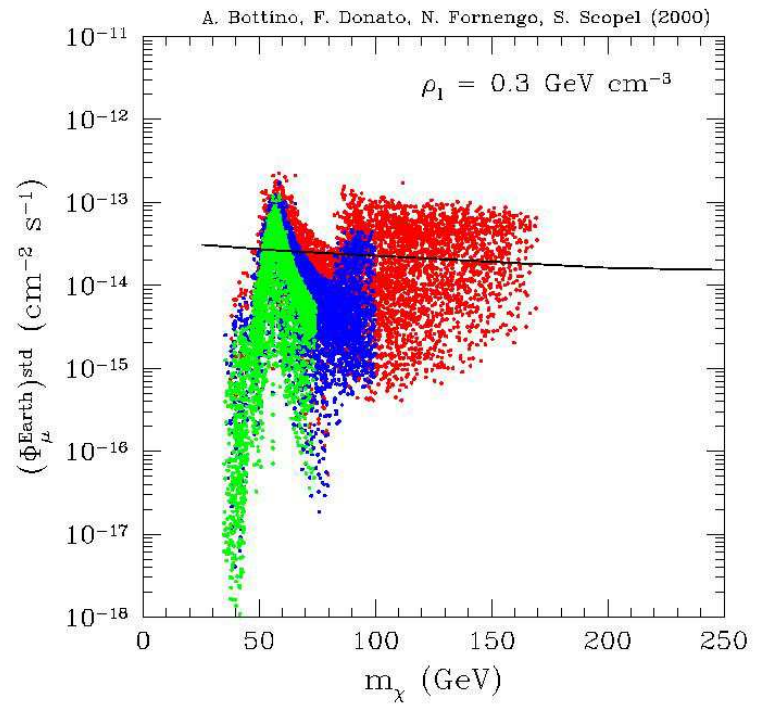

Figure 7. Flux of upgoing muons from neutralino annihilation inside the Earth in the effMSSM 52. The horizontal line is the upper bound from MACRO.

positrons 65, 66], antiprotons [56,65, 68] and antideuterium [67]. For this type of signals, global properties of the halo are relevant, and therefore in this case the matter distribution of neutralinos is an important quantity. In particular, the overdensities which would be present in a clumpy halo have the effect of largely enhancing the predicted signals [61, 65]. Neutrino and gamma fluxes require clumpiness to reach detectable levels. Also the recent anomaly in the HEAT data 85. on positrons may be explained by a signal originated by neutralino annihilation if overdensities are present in the galactic halo [66]. On the contrary, antiprotons and antideuterium may be detectable also in a smooth halo.

In fig. 9 we show the correlation between the antiproton and the antideuterium flux calculated in the effMSSM for a smooth halo 67. The vertical band represents the BESS 95+97 measurement of the antiproton flux. All the points on the right of this band have to be considered as excluded, since they provide a flux in excess of the measurements: this fact shows that the antiproton signal can be used to constrain the su-

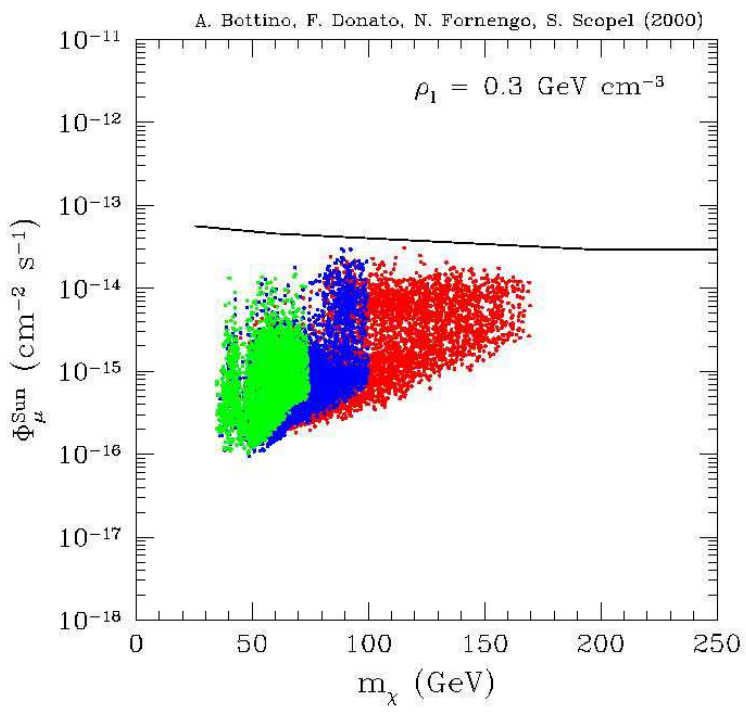

Figure 8. Flux of upgoing muons from neutralino annihilation inside the Sun in the effMSSM 52. The horizontal line is the upper bound from MACRO.

persymmetric parameter space. At present, there are no measurement of an antideuterium component in cosmic rays, but the analysis of Ref. 67 shows that the antideuterium signature is quite promising and the next generation detectors may be able to probe a sizeable fraction of supersymmetric configurations. This is shown in fig. 9 by the horizontal line, which denotes the reaching capability of the AMS detector on a 3-year flight on board of the space station. Recently, a detector designed specifically to look for antideuterium in cosmic rays has been proposed [86].

Finally, fig. 10 shows the predicted gammaray line flux in the effMSSM for a NFW halo, compared with an estimation of the GLAST sensitivity.

\section{Conclusions}

We have seen that we can identify two main issues in particle dark matter studies: i) to explain the observed amount of dark matter in the Universe $\left(0.05 \lesssim \Omega_{\mathrm{M}} h^{2} \lesssim 0.3\right)$ by finding suitable particle candidates; $\underset{i i}{ }$ ) to detect a relic particle. For both of them there appear to be good 


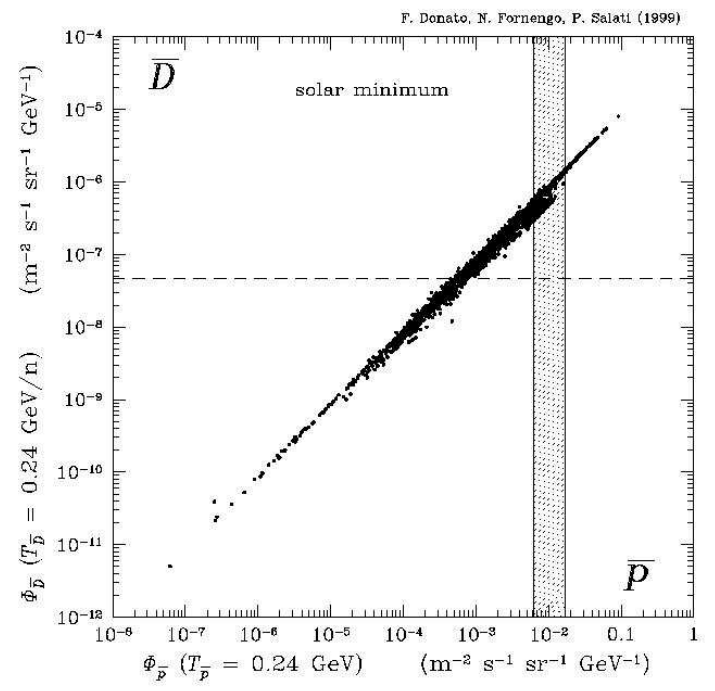

Figure 9. Flux of antideuterium vs. the flux of antiprotons from neutralino annihilation in the effMSSM for a smooth halo 67]. The vertical band is the measurement of BESS95+97. The horizontal line denotes the reaching capability of AMS on a 3-year flight on board of the space station

prospects of success.

As for the candidates, there are many proposed particles which could act as dark matter. Some of these candidates turn out to be quite natural, like e.g. the massive neutrino, the axion or the neutralino. Almost all of the proposed candidates can play the role of the dominant dark matter component, although for some of them a non-standard cosmology is required. An important remark is that, from the particle physics point of view, dark matter may naturally be multi-component. A multi-component dark matter scenario offers opportunity for interesting phenomenology not only to the dominant candidate, which would explain the cosmological observation on the $\Omega_{\mathrm{M}}$ parameter, but also to the sub-dominant candidates, since usually these are the ones which are easier to detect. The detection of a particle which is a relic from the early Universe would be a very important and exciting result.

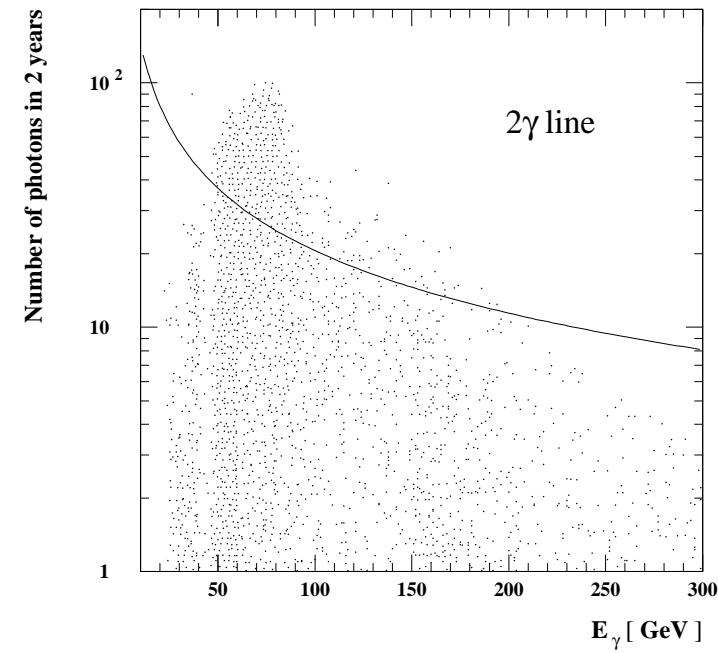

Figure 10. Predicted gamma-ray line flux in effMSSM for a NFW halo [63]. The solid line is the estimated GLAST sensitivity.

As for detection, perspectives are good, both for direct and for indirect detection techniques, especially for the most interesting and studied candidate, the neutralino. The possibility to have detectable rates for neutralinos depends on the specific supersymmetric model which is considered, and quite generally it appears simpler to detect a relic neutralino which is a sub-dominant dark matter component. Nevertheless, there are many supersymmetric schemes where relic neutralinos can provide enough cosmological abundance to explain the observed amount of dark matter, and at the same time they can have detection rates large enough to be accessible to direct, and also to some indirect, detection methods. The positive indication of annual modulation in the detection rate of the DAMA/NaI Collaboration can be interpreted as pointing towards a direct detection of a relic massive particle. This effect is, at the moment, the most compelling indication for a particle dark matter signal. When interpreted as originated from relic neutralinos, the annual modulation effect can be explained in a number of realization of supersymmetry. It is worth noticing that the presence of a signal from dark matter, like the annual modulation effect or 
signals which could hopefully come in future expeiments, can be very important not only for astrophysics and cosmology but also for particle physics, since the need to explain the effect can help in deriving properties of particle physics models and possibly discriminate among different realizations, for instance of supersymmetry.

Acknowledgements. This work was partially supported by the Research Grants of the Italian Ministero dell'Università e della Ricerca Scientifica e Tecnologica (MURST) within the Astroparticle Physics Project. I also wish to thank the Organizers for inviting me to deliver this talk at the TAUP 2001 Conference.

\section{REFERENCES}

1. See for instance: Y. Sofue and V. Rubin, astro-ph/0010594.

2. See, for instance: S.L. Bridle et al., astro$\mathrm{ph} / 9903472$

3. S. Perlmutter et al., Bull. Am. Astron. Soc. 29 (1997) 1351 astro-ph/9812473; S. Perlmutter et al., Astrophys. J. 517 (1999) 565 astro-ph/9812133; B. Schmidt et al., Astrophys. J. 507 (1998) 46; A.G. Riess, et al., Astrophys. J. 507 (1998) 46 astro-ph/9805200.

4. A. H. Jaffe et al. (Boomerang Collab.), Phys. Rev. Lett. 86 (2001) 3475 astro-ph/0007333; A. E. Lange et al. (Boomerang Collab.), Phys. Rev. D63 (2001) 042001 astro-ph/0005004; C. Pryke, N. W. Halverson, E. M. Leitch, J. Kovac, J. E. Carlstrom, W. L. Holzapfel and M. Dragovan, astro-ph/0104490.

5. P. de Bernardis et al, astro-ph/0105296.

6. See, for instance: S. Esposito, G. Mangano, G. Miele and O. Pisanti, Nucl. Phys. B568 (2000) 421 astro-ph/9906232.

7. A. Bottino and N. Fornengo, hep-ph/9904469

8. R. Rebolo, these Proceedings.

9. W. Freedman, these Proceedings.

10. See, for instance: N. Fornengo, M. C. Gonzalez-Garcia and J. W. Valle, Nucl. Phys. B580 (2000) 58 hep-ph/0002147; G.L. Fogli, E. Lisi and A. Marrone, Phys. Rev. D63 (2001) 053008 hep-ph/0009299.

11. E. Lisi, these Proceedings.

12. See, for instance: J.N. Bahcall, M.C.
Gonzalez-Garcia and C. Pena-Garay, hepph/0111150; G.L. Fogli, E. Lisi, D. Montanino, and A. Palazzo Phys. Rev. D62 (2000) 013002 hep-ph/9912231|; M.V. Garzelli and C. Giunti, hep-ph/0111254.

13. K. Enqvist, K. Kainulainen and J. Maalampi, Nucl.Phys.B317 (1989) 647.

14. K. Abazajian et al., Phys. Rev. D64 (2001) 023501 astro-ph/0101524; A.D. Dolgov and S.H. Hansen, Astropart. Phys. 16 (2002) 339 hep-ph/0009083.

15. See, for instance: E.W. Kolb and M.S. Turner, The Early Universe (AddisonWesley, 1988).

16. I. G. Irastorza, A. Morales and S. Scopel, hep$\mathrm{ph} / 0108146$.

17. T. Falk et al., Phys. Lett. B339 (1994) 248; L.J. Hall, T. Moroi and H. Murayama, Phys. Lett. B424 (1998) 305.

18. E.J. Chun, H.B. Kim and J.E. Kim, Phys. Rev. Lett. 72 (1994) 1956; A. Borgani, A. Masiero and M. Yamaguchi, Phys. Lett. B386 (1996) 189.

19. S. Dimopoulos, G.F. Giudice and A. Pomarol, Phys. Lett. B389 (1996) 37; T. Han and R. Hempfling, Phys. Lett. B415 (1997) 161.

20. S.A. Bonometto, F. Gabbiani and A. Masiero, Phys. Rev. D 49 (1994) 3918.

21. A. Kusenko and M. Shaposhnikov, Phys.Lett. B418 (1998) 46; A. Kusenko, V. Kuzmin, M. Shaposhnikov and P.G. Tinyakov, Phys. Rev. Lett. 80 (1998) 3185.

22. D.J.H. Chung, E.W. Kolb and A. Riotto, Phys. Rev. Lett. 81 (1998) 4048 hepph/9805473|; I.F.M. Albuquerque, L. Hui and E.W. Kolb, Phys. Rev. D64 (2001) 083504 hep-ph/0009017.

23. R.N. Mohapatra, V.L. Teplitz, Phys. Lett. B462 (1999) 302 astro-ph/9902085; R. N. Mohapatra, S. Nussinov and V. L. Teplitz, hep-ph/0111381.

24. A. Riotto, I. Tkachev, Phys. Lett. B484 (2000) 17 astro-ph/0003388; O. Seto, K. Kohri, T. Nakamura, JHEP 0109 (2001) 032 hep-ph/0109276; J.E. Lidsey, T. Matos, L.A. Urena-Lopez, astro-ph/0111292; M.C. Bento, O. B., R. Rosenfeld and L. Teodoro, Phys. Rev. D62, 041302, 2000 astro- 
ph/0101349]; M.C. Bento, O. Bertolami and R. Rosenfeld, Phys. Lett. B518, 276, 2001 hep-ph/0103340.

25. A. Bottino, F. Donato, N. Fornengo and S. Scopel, hep-ph/0105233.

26. V. Berezinsky, A. Bottino, J. R. Ellis, N. Fornengo, G. Mignola and S. Scopel, Astropart. Phys. 5 (1996) 1 [hep-ph/9508249].

27. V. Berezinsky, A. Bottino, J.R. Ellis, N. Fornengo, G. Mignola and S. Scopel, Astropart. Phys. 5 (1996) 333 hep-ph/9603342.

28. A. Bottino, F. Donato, N. Fornengo and S. Scopel, Phys. Rev. D59 (1999) 095004 hep$\mathrm{ph} / 9808459$.

29. A. Bottino, F. Donato, N. Fornengo and S. Scopel, Phys. Rev. D63 (2001) 125003 hep$\mathrm{ph} / 0010203$.

30. A. Bottino, N. Fornengo and S. Scopel, Nucl. Phys. B608 (2001) 461 [hep-ph/0012377].

31. J. Ellis, T. Falk, R. Ganis and K.A. Olive, Phys. Rev. D62 (2000) 075010 hepph/0004169|; J. Ellis, T. Falk, G. Ganis, K.A. Olive and M. Srednicki, Phys. Lett. B510 (2001) 236 hep-ph/0102098; J. Ellis and K.A. Olive, Phys. Lett. B514 (2001) 114 hep$\mathrm{ph} / 0105004$.

32. J. Ellis, S. Heinemeyer, K.A. Olive and G. Weiglein, Phys. Lett. B515 (2001) 348 hep$\mathrm{ph} / 0105061$.

33. J. Ellis, A. Ferst and K.A. Olive, hep$\mathrm{ph} / 0001005$.

34. J.L. Feng, K. Matchev and F. Wilczek, Phys. Lett. B482 (2000) 388 hep-ph/0004043; J.L. Feng, K. Matchev and T. Moroi, hepph/0003138; J.L. Feng and K. Matchev, Phys. Rev. D63 (2001) 095003 hep-ph/0011356.

35. E. Accomando, R. Arnowitt, B. Dutta and Y. Santoso, Nucl. Phys. B585 (2000) 124 hepph/0001019|; R. Arnowitt, B. Dutta and Y. Santoso, hep-ph/0008336.

36. T. Nihei, L. Roszkowski, R. Ruis de Austri, HEP 0105 (2001) 063 hep-ph/0102308; L. Roszkowski, R. Ruis de Austri, T. Nihei, HEP 0108 (2001) 024 hep-ph/0106334].

37. M.E. Gómez and J.D. Vegados, Phys. Lett. B512 (2001) 252 hep-ph/0012020.

38. A. Djouadi and M. Drees, Phys. Lett. B484 (2000) 183 hep-ph/0004205].
39. V. Mandic, A. Pierce, P. Gondolo and H. Murayama, hep-ph/0008022.

40. A.B. Lahanas, D.V. Nanopoulos and V.C. Spanos, Mod. Phys. Lett. A16 (2001) 1229 hep-ph/0009065.

41. J.L. Feng, K.T. Matchev and F. Wilczek, Phys. Rev. D63 (2001) 045024 astroph/0008115; V. Barger, F. Halzen, D. Hooper and C. Kao, hep-ph/0105182; V.A. Bednyakov, H.V. Klapdor-Kleingrothaus and H. Tu, Phys. Rev. D64 (2001) 075004 hepph/0101223 ; D. Hooper, Nucl. Phys. Proc. Suppl. 101 (2001) 347 hep-ph/0102011.

42. R. Jeannerot, X. Zhang and R. H. Brandenberger, JHEP 9912 (1999) 003 hepph/9901357]; W. B. Lin, D. H. Huang, X. Zhang and R. H. Brandenberger, Phys. Rev. Lett. 86 (2001) 954 astro-ph/0009003.

43. J. Ellis, A. Ferstl and K.A. Olive, Phys. Rev. D63 (2001) 065016 hep-ph/0007113]; J. Ellis and K.A. Olive, hep-ph/0106148.

44. A. Corsetti and P. Nath, hep-ph/0005234; A. Birkedal-Hansen and B. Nelson, Phys. Rev. D64 (2001) 015008 hep-ph/0102075].

45. M. Drees, Y.G. Kim and M.M. Nojiri, Phys. Rev. D63 (2001) 035008 hep-ph/0007202.

46. R. Arnowitt, B. Dutta and Y. Santoso, Nucl. Phys. B606 (2001) 59 [hep-ph/0102181].

47. G. Belanger, F. Boudjema, A. Pukhov and A. Semenov, hep-ph/0112278.

48. R. Arnowitt, B. Dutta and Y. Santoso, hepph/0005154; hep-ph/0101020.

49. A. Corsetti and P. Nath, Phys. Rev. D64 (2001) 125010 hep-ph/0003186.

50. E. Gabrielli, S. Khalil, C. Muñoz and E. Torrente-Lujan, Phys. Rev. D63 (2001) 025008 hep-ph/0006266; D.G. Cerdeño, S. Khalil and C. Muñoz, hep-ph/0105180.

51. D.G. Cerdeño, E. Gabrielli, S. Khalil, C. Muñoz and E. Torrente-Lujan, Nucl. Phys. B603 (2001) 231 hep-ph/0102270.

52. A. Bottino, F. Donato, N. Fornengo and S. Scopel, Phys. Rev. D62 (2000) 056006 hep$\mathrm{ph} / 0001309$.

53. A. Bottino, F. Donato, N. Fornengo and S. Scopel, Astropart. Phys. 13 (2000) 215 hep$\mathrm{ph} / 9909228$.

54. P. Belli, R. Bernabei, A. Bottino, F. Donato, 
N. Fornengo, D. Prosperi and S. Scopel, Phys. Rev. D61 (2000) 023512 hep-ph/9903501|.

55. A. Bottino, F. Donato, N. Fornengo and S. Scopel, Astropart. Phys. 10 (1999) 203 hep$\mathrm{ph} / 9809239$.

56. A. Bottino, F. Donato, N. Fornengo and P. Salati, Phys. Rev. D58 (1998) 123503 astro$\mathrm{ph} / 9804137$.

57. A. Bottino, F. Donato, N. Fornengo and S. Scopel, Phys. Rev. D59 (1999) 095003 hep$\mathrm{ph} / 9808456$.

58. A. Bottino, N. Fornengo, G. Mignola and L. Moscoso, Astropart. Phys. 3 (1995) 65 hep$\mathrm{ph} / 9408391$.

59. G. Duda, G. Gelmini and P. Gondolo, hepph/0102200.

60. V.A. Bednyakov and H.V. KlapdorKleingrothaus, Phys. Rev. D63 (2001) 095005 hep-ph/0011233].

61. P. Gondolo and J. Silk, Nucl. Phys. Proc. Suppl. 87 (2000) 87 hep-ph/0001070; P. Gondolo, Phys. Lett. B494 (2000) 181 hepph/0002226; G. Bertone, G. Sigl and J. Silk, Mon. Not. Roy. Astron. Soc. 326 (2001) 799 astro-ph/0101134; P. Ullio, H.S. Zhao and M. Kamionkowski, Phys. Rev. D64 (2001) 043504 astro-ph/0101481.

62. L. Bergström, J. Edsjö and C. Gunnarson, Phys. Rev. D63 (2001) 083515 astro-ph/0012346; L. Bergström, J. Edsjö and P. Ullio, Phys. Rev. Lett. 87 (2001) 251301 astro-ph/0105048; D.V. Hooper, hep-ph/0103277.

63. L. Bergström, P. Ullio and J. Buckley, Astropart. Phys. 9 (1998) 137 astro$\mathrm{ph} / 9712318$.

64. L. Bergström, J. Edsjö and P. Gondolo, astro$\mathrm{ph} / 9906033$.

65. L. Bergstrom, J. Edsjo, P. Gondolo and P. Ullio, Phys.Rev. D59 (1999) 043506 astro$\mathrm{ph} / 9806072$.

66. E.A. Baltz, J. Edsjo, K. Freese, P. Gondolo, astro-ph/0109318; G.L. Kane, Lian-Tao Wang and J.D. Wells, hep-ph/0108138.

67. F. Donato, N. Fornengo and P. Salati, Phys. Rev. D62 (2000) 043003 hep-ph/9904481.

68. L. Bergstrom, J. Edsjo and P. Ullio, astroph/9902012 J.D. Wells, A. Moiseev, J.F.
Ormes, Astrophys. J. 518 (1999) 570 hepph/9811325]; T. Mitsui, K. Maki and S. Orito, Phys. Lett. B389 (1996) 169 astro$\mathrm{ph} / 9608123$.

69. E.A. Baltz, C. Briot, P. Salati, R. Taillet and J. Silk, Phys. Rev. D61 (2000) 023514 astro$\mathrm{ph} / 9909112$.

70. K. Freese and P. Gondolo, Phys. Rev. D64 (2001) 123502 astro-ph/0106480.

71. S. Baek, P. Ko and Hong Seok Lee, Phys. Rev. D65 (2002) 035004 hep-ph/0103218.

72. T. Falk, hep-ph/0001207; P. Gondolo and K. Freeze, Nucl. Phys. Proc. Suppl. 87 (2000) 519 hep-ph/0001071; U. Chattopadhyay, T. Ibrahim and P. Nath, hep-ph/0005109.

73. B. Murakami, J.D. Wells, hep-ph/0011082; P. Ullio, JHEP 0106 (2001) 053 [hep$\mathrm{ph} / 010505]$.

74. I.M. Fisk and K. Nagai, http://www.ichep2000.hep.sci.osakau.ac.jp; P.J. Donan (ALEPH Collab.), http://alephwww.cern.ch/ALPUB/seminar/ lepc_mar2000/lepc2000.pdf.

75. J.A.Valls (CDF Collab.), http://fnalpubs. fnal.gov/archive/1999/conf/Conf-99-263E.html.

76. See, for instance: P. Gambino and M. Misiak, Nucl. Phys. B611 (2001) 338 hep$\mathrm{ph} / 0104034$.

77. R. Barate et al. (ALEPH Collab.) Phys. Lett. B495 (2000) 1 hep-ex/0011045; M. Acciarri et al. (L3 Collab.), Phys. Lett. B495 (2000) 18 hep-ex/0011043; ADLO Collabs. and the LEP Higgs Working Group, hep-ex/0107029.

78. G. Altarelli, F. Caravaglios, G.F. Giudice, P. Gambino and G. Ridolfi, JHEP 0106 (2001) 018 hep-ph/0106029].

79. J. Bijnens, E. Pallante and J. Prades, hepph/0112255; I. Blokland, A. Czarnecki and K. Melnikov, hep-ph/0112117; M. Hayakawa and T. Kinoshita, hep-ph/0112102; J. F. de Trocóniz and F. J. Ynduráin, hepph/0111258; A. Hocker, hep-ph/0111243; M. Knecht and A. Nyffeler, hep-ph/0111058; S. Narison, Phys.Lett. B513 (2001) 53 hep$\mathrm{ph} / 0103199$.

80. A. Morales, these Proceedings.

81. R. Bernabei et al. (DAMA Collab.), Phys. 
Lett. B480 (2000) 23; Eur. Phys. J. C18 (2000) 283.

82. A.K. Drukier, K. Freese and D.N. Spergel, Phys. Rev. D33, (1986) 3495; K. Freese, J. Frieman and A. Gould, Phys. Rev. D37 (1988) 3388.

83. S. Ambrosanio, A. Dedes, S. Heinemeyer, S. $\mathrm{Su}, \mathrm{G}$. Weiglein, hep-ph/0106255.

84. A. Bottino, F. Donato, N. Fornengo and S. Scopel, hep-ph/9710295.

85. S. W. Barwick et al. (HEAT Collab.), Astrophys. J. 482 (1997) L191; S. Coutu et al. (HEAT Collab.), Proc. of 27th ICRC (2001).

86. K. Mori et al., astro-ph/0109463. 